\title{
The Formation of Spherical Graphite in Cast Irons with the Economy of Rare Earth Metals

\author{
${ }^{1}$ German N. Safronov, ${ }^{2}$ Nikolay N. Safronov, ${ }^{3}$ Lenar R. Kharisov \\ ${ }^{1}$ Kazan Federal University \\ Email:ln271@mail.ru
}

Received: 23 ${ }^{\text {rd }}$ July 2019, Accepted: 10 $^{\text {th }}$ August 2019, Published: $31^{\text {st }}$ August 2019

\begin{abstract}
The article suggests an alternative technological process for obtaining high-strength cast irons, based on a synergetic complex of casting and metallurgical factors affecting cast iron in the stages of its melt preparation and crystallization in the mold, as well as resource-saving with use of the process of self-propagating high-temperature synthesis. The proposed process opens new prospects in the production of quality cast iron castings with the solution of problems of saving material and energy resources. Scientifically, these technological processes are the basis of a new paradigm compaction in graphitized cast irons without modifying its liquid state.
\end{abstract}

Keywords

High-Strength Cast Iron, Spherical Graphite, Compact Graphite, Modifying.

\section{Introduction}

Cast iron is the cheapest machine-building material with good casting properties, machinability with cutting, wear resistance, high resistance to alternating loads. All this causes the wide use of cast irons as a structural material. The main problem of cast irons, which determines their consumer qualities, is the morphology of graphite inclusions, which significantly affects the basic properties of cast iron, primarily on strength and ductility, characterizing cast iron as a structural material. Many of the operational properties of gray cast iron depend on the size and number of graphite inclusions. It is obvious that comparatively small inclusions with vortex plates with their uniform distribution ensure higher operating properties of cast iron. Large graphite plates with relatively straight pointed edges serve as sources of cracking, leading ultimately to the destruction of the part or product as a whole. In many large-sized inclusions of graphite, characteristic internal cracks are observed in the form of longitudinal discontinuities of continuity caused by growth stresses. A significant level of growth stress also serves as the reason for the pronounced loose package structure of the growth hillocks. The negative effect of graphite on the decrease in the indexes of mechanical properties decreases with increasing its compactness.

\section{Problem Statement}

Globular graphite weakens the metal matrix to the least extent, which leads to a sharp increase in the mechanical properties of cast iron with a purely pearlitic or bainitic structure, bringing their properties closer to the properties of carbon steels. The increased level of plasticity is provided with a purely ferrite matrix (in the cast or heat-treated state). High-strength cast iron has good casting and technological properties (fluidity, linear shrinkage, machinability with cutting), but it approaches steel by the value of concentrated volumetric shrinkage. Such cast iron is used to replace steel cast and forged parts (crankshafts of engines, compressors, etc.), as well as parts from malleable or conventional gray cast iron.

However, the technology of compacting the modification of liquid iron with producing graphite of vermicular and spherical shape in its structure, is not free of significant drawbacks, the main of which are: 1 use of modifiers, containing expensive and scarce elements of magnesium, cerium, yttrium, nickel, copper; 2 instability of the results of the process due to the strong dependence on the influence of scientific paradigm factors of the phenomenon of structural heredity [1]. The article suggests an alternative technology [2,3] of producing cast irons with compact graphite forms, which does not require graphitizing annealing or compacting modification of liquid iron and, as a result, is free from the above-mentioned drawbacks typical for these traditional technologies. The essence of the proposed technological process is based on the synergetic complex of foundry and metallurgical factors affecting cast iron in the stages of its melt preparation and crystallization in the mold. One of the components of this complex is the use, as charge materials, for preparing cast iron melt of disperse mechanical engineering waste containing oxidized iron (for example, forging scale). These waste products do not have any harmful impurities in their composition, such as S and P. Therefore, the cast iron obtained from them differs in purity with respect to the content of surface-active elements in them, which is an indispensable condition for the graphitization of cast iron in a compact form. In addition, the technology we have developed to produce a melt of cast iron from dispersed mechanical engineering waste takes it to the category of firstborn, that is, deprived of any hereditary signs, including negative ones, that prevent the formation of compact forms of crystallizable graphite.

This technology of synthesizing cast iron melt also provides for its overheating, which contributes to homogenization and, as a result, supercooling during the transition to the solid phase. This circumstance is also a compacting factor in the formation of graphite inclusions in the metallic matrix of cast iron. The conditions for the formation of a melt of cast 
iron described above satisfy the thermodynamic criterion of compact graphitization in the form of the chemical potential of carbon in carbide-type ordering. According to this criterion, compact graphitization is more likely, the higher the melt overheating and the higher its propensity to metastable crystallization. In this case, conditions are created for the formation of a carbide-shaped fluctuation in the melt, in which the carbon activity determines a sufficiently high thermodynamic graphitization potential. The magnitude of the latter depends on a number of conditions: the presence of third elements that form quasi- chemical orderings in the melt with iron and carbon; temperature of melt overheating; the degree of its refining by surfactants. In our case, the creation and "discharge" of the thermodynamic potential of compact graphitization is due to superheating of the melt into the supercritical state, in which a potential of compact graphitization is created on the basis of the formation of ordered macrozones with pseudocarbide stoichiometry. The destruction of these zones, which leads to the formation of point graphite, a building material for the formation of compact graphite inclusions, is achieved by an optimal regime of supercooling and crystallization of highly overheated and deeply refined melts.

\section{Experimental Results and Discussion}

We also proposed a technological process for producing cast irons with compact graphite forms [4], which cardinally solves the problem of energy saving, since the issue of providing energy from outside has ceased to exist. The selfpropagating high-temperature synthesis (SHS) is based on the technological process under discussion, in which the necessary amount of released energy for the process, the transfer of solid materials into the liquid superheated state, is incorporated in the charge materials. In this case, the charge is completely made up of disperse waste of engineering (forge scale, aluminum and graphite chips, used abrasive tools made of silicon carbide). Forge scale, containing about $70 \%$ of oxidized iron, which, being recovered, is the basis of cast iron. As a reducing agent, it is proposed to use siluminous chips, which along with a strong reducing agent (Al) contains a basic alloying element in Si cast iron. Thus, the combination: forge scale - silumin shaving in a certain relationship creates the prerequisites for obtaining cast iron from these dispersed wastes of mechanical engineering by implementing between the components of the SHS process. For the implementation of the SHS process, the charge mixture was made with the following ratio of components: forge scale / silumin chips (AK18) / ammonium nitrate / chalk / graphite chips $=100 / 57 / 62 / 21 / 0,5$. The components of the charge were thoroughly mixed and placed in a graphite cylinder that rotated in such a way that the charge and products of the process were subjected to centrifugal force. For the creation of artificial gravity with a coefficient, the formula for selecting the rotational speed ( $\mathrm{n}, \mathrm{rpm})$ of the graphite cylinder was used:

$$
n=\frac{300 \sqrt{k}}{r},
$$

where: $r$ - is the radius of rotation of the graphite cylinder.

Figure 1 shows the microstructure of the heat-resistant cast iron of the brand ChS5SH, synthesized according to our proposed technology. The metallic matrix is represented by siliceous ferrite with small sections of perlite. Inclusions of graphite have a compact and dotted form. The formation of compact graphite in the structure of cast iron is due to high rates of heating, overheating and cooling of liquid SHS-cast iron, its alloying with magnesium and minimization in the composition of surface-active elements.

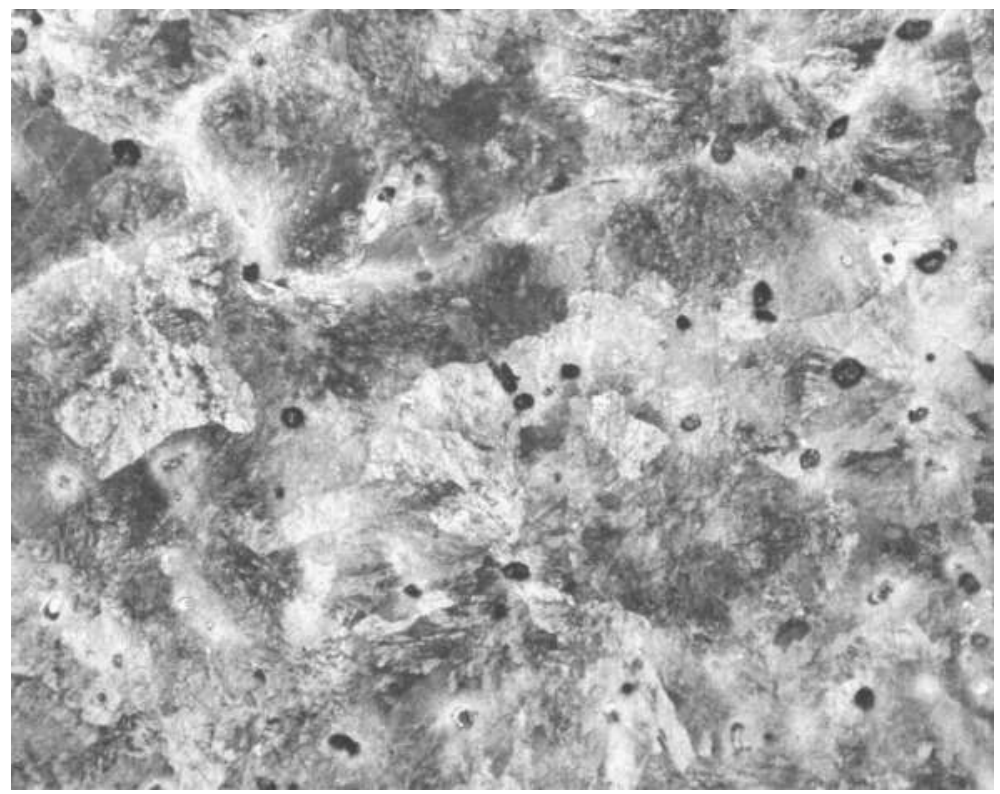

Figure 1: Microstructure of SHS-Cast Iron, $\times 200$ 
The characteristic feature of the conditions for the crystallization of SHS-cast iron is the presence of increased supercooling due to both the homogenization of the melt and the high thermal activity of the graphite form. We carried out the investigations of the crystallization of SHS-cast iron by the thermal analysis method, which was carried out using a multi-point automatic system (AIST), which allows recording the cooling curves and differential curves dT/dt. The thermocouple BP 5/20 was used as the temperature sensor. It was found that the crystallization processes proceed with supercooling equal to 6.2 degrees. When the SHS process under conditions of artificial gravitation is carried out, a higher supercooling value should be expected due to the temperature increase of the iron crystallization with increasing pressure [5].

The SHS process of SHS cast iron is composed in such a way that, along with forge scale, an ammonia nitrate is also used as an oxidizing agent. Alumina and gaseous reaction products containing nitrogen are formed upon its restoration. It is known [6] that during the course of the SHS process in the gas phase on the basis of nitrogen, nitrides, carbides and carbonitrides are formed under the condition that elements containing an affinity for nitrogen and carbon are present in the charge. In our case, such elements are aluminum and silicon. These refractory particles present in the metallic phase have a size of several nm [7] and act as numerous centers of crystallization as structural constituents of the metal matrix [8] and graphite inclusions.

\section{Conclusions}

Thus, the proposed technological processes for the production of graphitized cast irons, which promote the formation of compact graphite forms without modifying processing of the liquid state, open new prospects in the production of quality cast iron castings with the solution of problems of saving material and energy resources. Scientifically, these technological processes are the basis of a new paradigm - compaction in graphitized cast irons without modifying of its liquid state.

\section{Acknowledgements}

The work is performed according to the Russian Government Program of Competitive Growth of Kazan Federal University.

\section{References}

[1] Nikitin V.I., Nikitin K.V. Heredity in cast alloys. - Moscow: Mechanical Engineering - 2005. - 476 p.

[2] Bikulov R.A., Safronov N.N. Technological process of guaranteed compact graphitization of cast irons // Foundry of Russia. - 2010. - No. 1. - P. 34-36.

[3] Bikulov R.A., Safronov N.N. Method for the production of aluminum cast iron with compact graphite inclusions RU 2487950 C1, IPC C21C 1/10 (2006.01)

[4] Physical quantities: Reference book / A.P. Babichev, N.A. Babushkina, A.M. Bratkovsky and others; Ed. I.S. Grigorieva, E.Z. Meilikhova. - M .: Energoatomizdat, 1991. - 1232 p.

[5] Safronov G.N., Safronov N.N., Kharisov L.R. SHS ferroaluminum obtained from the disperse waste of engineering //Material Science Forum. Volume 870. - 2016. - P.437-440. DOI: 10.4028/www.scientific.net/MSF.870.437

[6] Korchinsky M. Advanced metal structural materials and the new role of microalloyed steels // Steel. - 2005. № 6. P. 124-130.

[7] Chumanov V.I., Chumanov I.V., Anikeev A.N., Garifulin R.R. Strengthening of surface layers during the formation of hollow billets by the method of centrifugal casting // Elektrometallurgiya. - 2010. № 1. - P. 33-36.

[8] Makarenko K.V. Control of the processes of structurization in castings of alloyed gray cast iron, News of High schools. Ferrous metallurgy. - 2010. No. 1. - P. 45-49. 